\title{
Pentagonal Graph for Designing Manufacturing Cellular System
}

\section{S Thirunavukkarasu}

\begin{abstract}
In this paper we have analyzedthat pentagonal snake PSk, subdivision of a pentagonal snake S(PSk) and alternate pentagonal snake $A(P S k)$ are mean square cordial graphs.
\end{abstract}

Keywords: Mean square cordial labeling, Pentagonalsnake, Subdivision of a pentagonal snake,alternative pentagonal snake.

\section{INTRODUCTION}

One of the most engrossing and booming areas of mathematics is graph theory which is an analysis of graph that deals with the relationship of vertices and edges. Due to the involvement of the researchers for the past 60 years, over 200 graphs labeling techniques [1] have been discussed in thousands of research papers. Graph with labeling serves as a useful model with greater number of applications like the concepts of coding, study of crystals, radar detections, astro studies, designing circuits, communication network addressing, managing data bases, sharing secret messages and it simulates many constrained programming in finite number of domains. Along with these applications, this technique is applied in disk redundancies, manufacturing design in drilling machines, circuit board designs, network configuration etc Here Harary[2] is followed for basic notations.Cordial labeling was introduced by Cahit[3] and Ponraj etal[4] wereinitiated the mean cordial labeling of a graph .Mean square cordial labeling introduced by A.Nellai murugan etal and they have discussed it for some special graphs[5].Moreover they have discussed the mean square cordial labeling for some tree and cycle related graphs[6,7]. Dhanalakshmi etal have discussed mean square cordial labeling related to some cyclic and acyclic graphs and its rough approximations $[8,9]$. In this paper we analysed that pentagonal snake $\mathrm{PS}_{\mathrm{k}}$, subdivision of a pentagonal snake $\mathrm{S}\left(\mathrm{PS}_{\mathrm{k}}\right)$ and alternate pentagonal snake $\mathrm{A}\left(\mathrm{PS}_{\mathrm{k}}\right)$ are mean square cordial graphs.

\section{PRELIMINARIES}

Definition 1: Let $G=(V, E)$ be a graph with $p$ vertices and q edges. "A Mean Square Cordial labeling of a Graph G(V, E) with $p$ vertices and $q$ edges is a bijection from $V$ to $\{0$, 1) such that each edge uv is assigned the label $\left(\left|\left(f(u)^{2}+f(v)^{2}\right) / 2\right|\right)$ where $\lceil x\rceil$ (ceil $\left.(\mathrm{x})\right)$ is the least integer greater than or equal to $\mathrm{x}$ with the condition that the

Revised Manuscript Received on December 11, 2019

S.Thirunavukkarasu, Department of Information Technology, Bharath Institute of Higher Education and Research, Tambaram, India. number of vertices labeled with 0 and the number of vertices labeled with 1 differ by at most 1 and the number of edges labeled with 0 and the number of edges labeled 0 and the number of edges labeled with 1 differ by at most 1 "'.

Definition 2: The pentagonal snake $P\left(S_{k}\right)$ is obtained from a path $\mathrm{u}_{1}, \mathrm{u}_{2}, \ldots . \mathrm{u}_{\mathrm{k}}$ by joining $\mathrm{u}_{\mathrm{i}}$ and $\mathrm{u}_{\mathrm{i}+1}$ for $\quad 1 \leq \mathrm{i}$ $\leq \mathrm{k}-1$, to two new vertices $\mathrm{v}_{\mathrm{i}}, \mathrm{w}_{\mathrm{i}}, \mathrm{x}_{\mathrm{i}}$ and then joining $\mathrm{v}_{\mathrm{i}}, \mathrm{x}_{\mathrm{i}}$ and $\mathrm{x}_{\mathrm{i}}, \mathrm{w}_{\mathrm{i}}$. That is the path $\mathrm{P}_{\mathrm{n}}$ by replacing each edge of the path by a cycle $\mathrm{C}_{5}$.

Definition 3: Let $G$ be a graph. The subdivision graph $S(G)$ is obtained from $G$ by subdividing each edge of $G$ with a vertex

Definition 4: An alternate pentagonal snake $\mathrm{A}\left(\mathrm{PS}_{\mathrm{k}}\right)$ is obtained from a path $\mathrm{u}_{1}, \mathrm{u}_{2}, \ldots, \mathrm{u}_{\mathrm{n}}$ by joining $\mathrm{u}_{\mathrm{i}}$ and $\mathrm{u}_{\mathrm{i}+1}$ to two new vertices $v_{i}, w_{i}$ and by joining $v_{i}$ and $w_{i}$ to a new vertex $\mathrm{x}_{\mathrm{i}}$ respectively. That is, every alternate edge of a path is replaced by a cycle $\mathrm{C}_{5}$

\section{MAIN RESULTS}

Theorem 1 Pentagonal snake $\mathrm{PS}_{\mathrm{k}}$ admits mean square cordial labeling $\forall \mathbf{k} \geq 2$.

Proof: Let Pkbe the path $\mathrm{u}_{1}, \mathrm{u}_{2}, \ldots . \mathrm{u}_{\mathrm{k}}$. Let $\mathrm{V}\left(\mathrm{PS}_{\mathrm{k}}\right)=$ $\mathrm{V}\left(\mathrm{P}_{\mathrm{k}}\right) \cup\left\{\mathrm{v}_{\mathrm{i}}, \mathrm{w}_{\mathrm{i}}, \mathrm{X}_{\mathrm{i}}: \mathrm{i}\right.$ varies from 1 to $\left.\mathrm{k}-1\right\}$ and $\mathrm{E}\left(\mathrm{PS}_{\mathrm{k}}\right)=\left\{\left[\left(\mathrm{u}_{\mathrm{i}} \mathrm{u}_{\mathrm{i}+1}\right): \mathrm{i}\right.\right.$ varies from 1 to $\left.\mathrm{k}-1\right] \cup\left[\left(\mathrm{u}_{\mathrm{i}} \mathrm{v}_{\mathrm{i}}\right.\right.$ : $\mathrm{i}$ varies from 1 to $\mathrm{k}-1\} \cup\left[\left(\mathrm{v}_{\mathrm{i}} \mathrm{x}_{\mathrm{i}}: \mathrm{i}\right.\right.$ varies from 1 to $\left.\mathrm{k}-1\right] \cup\left[\left(\mathrm{x}_{\mathrm{i}} \mathrm{W}_{\mathrm{i}}: \mathrm{i}\right.\right.$ varies from 1 to $\mathrm{k}-1\} \cup\left[\left(\mathrm{w}_{\mathrm{i}} \mathrm{u}_{\mathrm{i}}: \mathrm{i}\right.\right.$ varies from 1 to $\left.\mathrm{k}-1\right]$ Here

$$
|V|=4 k-3 \text { and }|E|=5 k-5
$$

Define f maps $\mathrm{V}\left(\mathrm{PS}_{\mathrm{k}}\right)$ to $\{0,1\}$

Case (i) $\mathrm{k}$ is odd

$$
\begin{aligned}
f\left(v_{i}\right)= & 0, i \text { var ies from } 1 \text { to }(k-1) / 2 \\
& 1, i \text { var ies from }(k+1) / 2 \text { to } k-1 \\
f\left(u_{i}\right)= & 0, i \text { var ies from } 1 \text { to }(k+1) / 2 \\
& 1, i \text { var ies from }(k+3) / 2 \text { to } k \\
f\left(w_{i}\right)= & 0, i \text { var ies from } 1 \text { to }(k-1) / 2 \\
& 1, i \text { var ies from }(k+1) / 2 \text { to } k-1 \\
f\left(x_{i}\right)= & 0, i \text { var ies from } 1 \text { to }(k-1) / 2 \\
& 1, i \text { var ies from }(k+1) / 2 \text { to } k-1
\end{aligned}
$$$$
\text { The }
$$

Therfallewidgced edge labeling is as follows 


\section{Pentagonal Graph For Designing Manufacturing Cellular System}

$$
\begin{aligned}
& f\left(u_{i} u_{i+1}\right)=0, i \text { var ies from } 1 \text { to }(k-1) / 2 \\
& 1, i \text { var ies from }(k+1) / 2 \text { to } k-1 \\
& f\left(u_{i} v_{i}\right)=0, i \text { var ies from } 1 \text { to }(k-1) / 2 \text {, } \\
& 1, i \text { var ies from }(k+1) / 2 \text { to } k-1 \text {, } \\
& f\left(v_{i} x_{i}\right)=0, i \text { var ies from } 1 \text { to }(k-1) / 2 \\
& 1, i \text { var ies from }(k+1) / 2 \text { to } k-1 \text {, } \\
& f\left(x_{i} w_{i}\right)=0, i \text { var ies from } 1 \text { to }(k-1) / 2 \\
& 1, i \text { var ies from }(k+1) / 2 \text { to } k-1 \text {, } \\
& f\left(u_{i+1} w_{i}\right)=0, i \text { var ies from } 1 \text { to }(k-1) / 2 \\
& 1, i \text { var ies from }(k+1) / 2 \text { to } k-1 \text {, }
\end{aligned}
$$

The following table expresses the cardinality of vertices and edges of MSCL for the above graph.

\begin{tabular}{|c|c|l|l|}
\hline \multicolumn{2}{|c|}{$\mathrm{T}$} & 0 & 1 \\
\hline$\left|v_{f}(t)\right|$ & $2 k-1$ & $2 k-2$ \\
\hline$\left|e_{f}(t)\right|$ & $\frac{5 k-5}{2}$ & $\frac{5 k-5}{2}$ \\
\hline
\end{tabular}

\section{Case (ii) $k$ is even}

$$
\begin{aligned}
f\left(u_{i}\right)= & 0, i \text { var ies from } 1 \text { to } k / 2 \\
& 1, i \text { var ies from }(k+2) / 2 \text { to } k \\
f\left(v_{i}\right)= & 0, i \text { var ies from } 1 \text { to }(k-1) / 2 \\
& 1, i \text { var ies from }(k+1) / 2 \text { to } k-1 \\
f\left(w_{i}\right)= & 0, i \text { var ies from } 1 \text { to }(k-2) / 2 \\
& 1, i \text { var ies from } k / 2 \text { to } k-1 \\
f\left(x_{i}\right)= & 0, i \text { var ies from } 1 \text { to }(k-1) / 2 \\
& 1, i \text { var ies from }(k+1) / 2 \text { to } k-1
\end{aligned}
$$

Then the induced edge labeling is as follows

$$
\begin{aligned}
& f\left(u_{i} u_{i+1}\right)=0, i \text { var ies from } 1 \text { to }(k-2) / 2 \\
& 1, i \text { var ies from } k / 2 \text { to } k-1 \\
& f\left(u_{i} v_{i}\right)=0, i \text { var ies from } 1 \text { to } k / 2 \text {, } \\
& 1, i \text { var ies from }(k+2) / 2 \text { to } k-1 \text {, } \\
& f\left(v_{i} x_{i}\right)=0, i \text { var ies from } 1 \text { to } k / 2 \\
& 1, i \text { var ies from }(k+2) / 2 \text { to } k-1 \text {, } \\
& f\left(x_{i} w_{i}\right)=0, i \text { var ies from } 1 \text { to }(k-2) / 2 \\
& 1, i \text { var ies from } k / 2 \text { to } k-1 \text {, } \\
& f\left(u_{i+1} w_{i}\right)=0, i \text { var ies from } 1 \text { to }(k-2) / 2 \\
& 1, i \text { var ies from } k / 2 \text { to } k-1 \text {, }
\end{aligned}
$$

The Following Table Expresses The Cardinality Of Vertices And Edges Of Mscl For The Above Graph.

\begin{tabular}{|c|l|l|}
\hline $\mathrm{T}$ & 0 & 1 \\
\hline$\left|v_{f}(t)\right|$ & $2 k-1$ & $2 k-2$ \\
\hline
\end{tabular}

\begin{tabular}{|c|c|c|}
\hline $\mathrm{T}$ & 0 & 1 \\
\hline$\left|\mathrm{p}_{\alpha}(\mathrm{t})\right|$ & $\frac{9 k-7}{2}$ & $\frac{9 k-9}{2}$ \\
\hline $\mid a_{y}\left(\mathbb{L}^{2}\right)$ & $5 k-5$ & $5 k-5$ \\
\hline
\end{tabular}

\begin{tabular}{|l|c|c|}
\hline$\left|e_{f}(t)\right|$ & $\frac{5 k-6}{2}$ & $\frac{5 \mathrm{k}-4}{2}$ \\
\hline
\end{tabular}

Hence pentagonal snake $\mathrm{PS}_{\mathrm{k}}$ admits mean square cordial labeling $\forall \mathrm{k} \geq 2$.

Illustration:

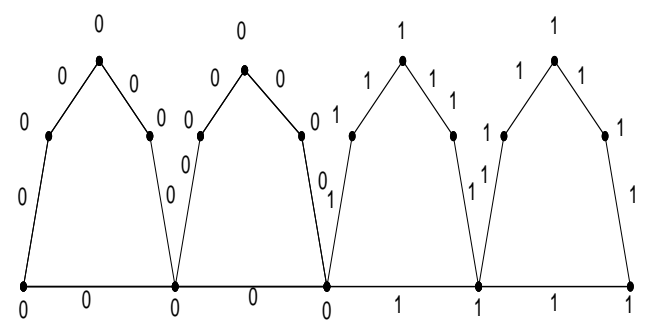

Figure 1:Mean square cordial labeling of pentagonal snake PS5

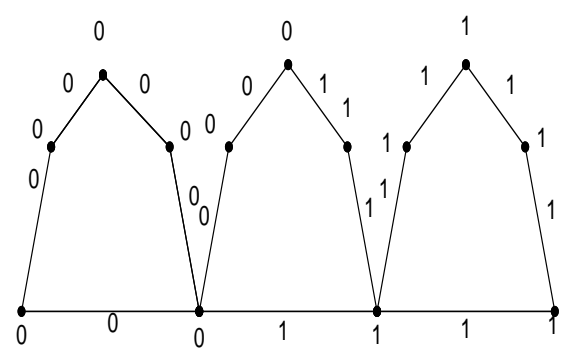

Figure 2:Mean square cordial labeling of pentagonal snake $\mathrm{PS}_{4}$

Theorem: 2 Subdivision of a pentagonal snake $\mathbf{S}\left(\mathbf{P S}_{\mathrm{k}}\right)$ admits mean square cordial labeling $\forall k \geq 3$ and $k$ is odd.

Proof: Let Pkbe the path $\mathrm{u}_{1}, \mathrm{u}_{2}, \mathrm{u}_{\mathrm{k}}$. Let $\mathrm{V}\left(\mathrm{PS}_{\mathrm{k}}\right)=$ $\mathrm{V}\left(\mathrm{P}_{\mathrm{k}}\right) \cup\left\{\mathrm{v}_{\mathrm{i}}, \mathrm{w}_{\mathrm{i}}, \mathrm{x}_{\mathrm{i}}\right.$ : varies from 1 to $\left.\mathrm{k}-1\right\}$ and $\mathrm{V}\left(\mathrm{S}\left(\mathrm{PS}_{\mathrm{k}}\right)\right)=$ $\mathrm{V}\left(\mathrm{PS}_{\mathrm{k}}\right) \cup\left\{\mathrm{a}_{\mathrm{i}}, \mathrm{b}_{\mathrm{i}}, \mathrm{c}_{\mathrm{i}}, \mathrm{d}_{\mathrm{i}}, \mathrm{e}_{\mathrm{i}} \mathrm{i}\right.$ varies from 1 to $\left.\mathrm{k}-1\right\}$ Then $\mathrm{E}\left(\mathrm{S}\left(\mathrm{PS}_{\mathrm{k}}\right)\right)=\left\{\left[\left(\mathrm{u}_{\mathrm{i}} \mathrm{b}_{\mathrm{i}}\right)\right.\right.$ : i varies from 1 to $\left.\mathrm{k}-1\right] \cup\left[\left(\mathrm{b}_{\mathrm{i}} \mathrm{v}_{\mathrm{i}}\right)\right.$ : i varies from 1 to $\mathrm{k}-1] \cup\left[\left(\mathrm{v}_{\mathrm{i}} \mathrm{c}_{\mathrm{i}}: \mathrm{i}\right.\right.$ varies from 1 to $\left.\mathrm{k}-1\right\} \cup\left[\left(\mathrm{c}_{\mathrm{i}} \mathrm{x}_{\mathrm{i}}: \mathrm{i}\right.\right.$ varies from 1 to $\mathrm{k}-1] \cup\left[\left(\mathrm{x}_{\mathrm{i}} \mathrm{d}_{\mathrm{i}}\right.\right.$ :i varies from 1 to $\mathrm{k}-1] \cup\left[\left(\mathrm{d}_{\mathrm{i}} \mathrm{w}_{\mathrm{i}}\right.\right.$ : $\mathrm{i}$ varies from 1 to $\mathrm{k}-1 \cup\left[\left(\mathrm{w}_{\mathrm{i}} \mathrm{e}_{\mathrm{i}}\right.\right.$ : $\mathrm{i}$ varies from 1 to $\mathrm{k}-1 \cup\left[\left(\mathrm{e}_{\mathrm{i}} \mathrm{u}_{\mathrm{i}+1}: \mathrm{i}\right.\right.$ varies from 1 to $\left.\mathrm{k}-1\right] \cup\left[\left(\mathrm{a}_{\mathrm{i}} \mathrm{u}_{\mathrm{i}+1}\right.\right.$ : $\mathrm{i}$ varies from 1 to $\mathrm{k}-1\} \cup\left[\left(\mathrm{a}_{\mathrm{i}} \mathrm{u}_{\mathrm{i}}\right.\right.$ : $\mathrm{i}$ varies from 1 to $\left.\mathrm{k}-1\right\}$

Here $|V|=9 k-8$ and $|E|=10 k-10$

Define $\mathrm{f}$ maps $\mathrm{V}\left(\mathrm{S}\left(\mathrm{PS}_{\mathrm{k}}\right)\right)$ to $\{0,1\}$ 


$$
\begin{aligned}
& f\left(u_{i}\right)=0, i \text { var ies from } 1 \text { to }(k+1) / 2 \\
& 1, i \text { var ies from }(k+3) / 2 \text { to } k \\
& f\left(v_{i}\right)=0, i \text { var ies from } 1 \text { to }(k-1) / 2 \\
& 1, i \text { var ies from }(k+1) / 2 \text { to } k \\
& f\left(w_{i}\right)=0, i \text { var ies from } 1 \text { to }(k-1) / 2 \\
& 1, i \text { var ies from }(k+1) / 2 \text { to } k \\
& f\left(x_{i}\right)=0, i \text { var ies from } 1 \text { to }(k-1) / 2 \\
& 1, i \text { var ies from }(k+1) / 2 \text { to } k-1 \\
& f\left(a_{i}\right)=0, i \text { var ies from } 1 \text { to }(k-1) / 2 \\
& 1, i \text { var ies from }(k+1) / 2 \text { to } k-1 \\
& f\left(b_{i}\right)=0, i \text { var ies from } 1 \text { to }(k-1) / 2 \\
& 1, i \text { var ies from }(k+1) / 2 \text { to } k-1 \\
& f\left(c_{i}\right)=0, i \text { var ies from } 1 \text { to }(k-1) / 2 \\
& 1, i \text { var ies from }(k+1) / 2 \text { to } k-1 \\
& f\left(d_{i}\right)=0, i \text { var ies from } 1 \text { to }(k-1) / 2 \\
& 1, i \text { var ies from }(k+1) / 2 \text { to } k-1 \\
& f\left(e_{i}\right)=0, i \text { var ies from } 1 \text { to }(k-1) / 2 \\
& 1, i \text { var ies from }(k+1) / 2 \text { to } k-1
\end{aligned}
$$

Then the induced edge labeling is as follows

$$
\begin{aligned}
f\left(u_{i} b_{i}\right)= & \begin{array}{r}
0, i \text { var ies from } 1 \text { to }(k-1) / 2, \\
1, i \text { var ies from }(k+1) / 2 \text { to }
\end{array} \\
f\left(b_{i} v_{i}\right)= & 0, i \text { var ies from } 1 \text { to }(k-1) / 2 \\
& 1, i \text { var ies from }(k+1) / 2 \text { to } k
\end{aligned}
$$

$$
\begin{array}{r}
f\left(e_{i} u_{i+1}\right)= \\
1, i \text { var ies from }(k+1) / 2 \text { to } k \\
\begin{aligned}
& f\left(u_{i+1} a_{i}\right)= 0, i \text { var ies from } 1 \text { to }(k-1) / \\
& 1, i \text { var ies from }(k+1) / 2 \text { to } k
\end{aligned} \\
\begin{aligned}
f\left(a_{i} u_{i}\right)= & 0, i \text { var ies from } 1 \text { to }(k-1) / 2, \\
& 1, i \text { var ies from }(k+1) / 2 \text { to } k .
\end{aligned}
\end{array}
$$

The following table expresses the cardinality of vertices and eges of MSCL for the above graph.

Hence subdivision of a pentagonal snake $\mathrm{S}\left(\mathrm{PS}_{\mathrm{k}}\right)$ admits mean square cordial labeling $\forall k \geq 2$.

Illustration:

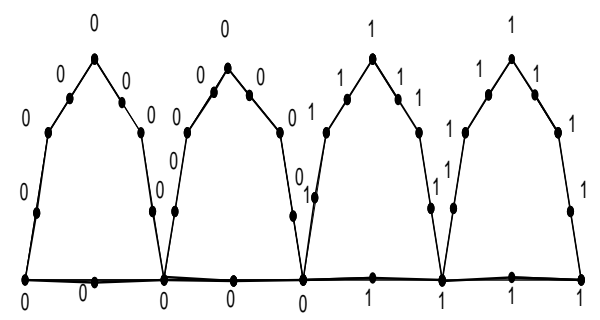

Figure 3:Mean square cordial labeling of pentagonal snake PS5

Theorem: 3 Mean square cordial labeling of a alternate pentagonal snakeA $\left(\mathrm{PS}_{2 \mathrm{k}}\right)$,

$k \geq 2$ where the pentagon starts from $u_{1}$ and ends with $\mathbf{u}_{2 \mathrm{k}}$.

Proof: Let Pkbe the path $\mathrm{u}_{1}, \mathrm{u}_{2}, . . \mathrm{u}_{2 \mathrm{k}}$. Let $\mathrm{V}\left(\mathrm{A}\left(\mathrm{PS}_{\mathrm{k}}\right)\right)=$ $\mathrm{V}\left(\mathrm{P}_{\mathrm{k}}\right) \cup\left\{\mathrm{v}_{\mathrm{i}}, \mathrm{w}_{\mathrm{i}}, \mathrm{x}_{\mathrm{i}}: \mathrm{i}\right.$ varies from 1 to $\left.\mathrm{k}-1\right\}$ and $\mathrm{E}\left(\mathrm{A}\left(\mathrm{PS}_{\mathrm{k}}\right)\right)=\left\{\left[\left(\mathrm{u}_{\mathrm{i}} \mathrm{u}_{\mathrm{i}+1}\right): \mathrm{i}\right.\right.$ varies from 1 to $\left.\mathrm{k}-1\right] \cup\left[\left(\mathrm{u}_{2 \mathrm{i}} \mathrm{u}_{2 \mathrm{i}+1}\right): \mathrm{i}\right.$ varies from $\mathrm{k}$ to $\mathrm{k}-1] \cup\left[\left(\mathrm{u}_{\mathrm{i}} \mathrm{v}_{\mathrm{i}}\right.\right.$ : $\mathrm{i}$ varies from 1 to $\left.\mathrm{k}-1\right\} \cup\left[\left(\mathrm{v}_{\mathrm{i}} \mathrm{x}_{\mathrm{i}}\right.\right.$ $: \mathrm{i}$ varies from 1 to $\mathrm{k}-1] \cup\left[\left(\mathrm{x}_{\mathrm{i}} \mathrm{w}_{\mathrm{i}}\right.\right.$ : $\mathrm{i}$ varies from 1 to $\left.\mathrm{k}-1\right\} \cup$ $\left[\left(\mathrm{w}_{\mathrm{i}} \mathrm{u}_{\mathrm{i}+1}: \mathrm{i}\right.\right.$ varies from 1 to $\left.\mathrm{k}-1\right]$ Here

$|V|=5 k$ and $|E|=6 k-1$

Define f mapsV $\left(\mathrm{A}\left(\mathrm{PS}_{\mathrm{k}}\right)\right)$ to $\{0,1\}$

Case (i) $k$ is odd

$$
\begin{aligned}
f\left(u_{i}\right)= & 0, i \text { var ies from } 1 \text { to }(2 k) / 2 \\
& 1, i \text { var ies from }(2 k+2) / 2 \text { to } 2 k
\end{aligned}
$$

$$
\begin{aligned}
f\left(v_{i}\right)= & 0, i \text { var ies from } 1 \text { to }(k+1) / 2 \\
& 1, i \text { var ies from }(k+3) / 2 \text { to } k
\end{aligned}
$$

$$
f\left(x_{i}\right)=0, i \text { var ies from } 1 \text { to }(k-1) / 2
$$$$
1, i \text { var ies from }(k+1) / 2 \text { to } k
$$ 


\section{Pentagonal Graph For Designing Manufacturing Cellular System}

Then the induced edge labeling is as follows

$$
\begin{aligned}
& f\left(u_{i} u_{i+1}\right)=0, i \text { var ies from } 1 \text { to }(2 k) / 2 \\
& 1, i \text { var ies from }(2 k+2) / 2 \text { to } 2 k-1
\end{aligned}
$$

$$
\begin{array}{r}
f\left(u_{i} v_{i}\right)=0, i \text { var ies from } 1 \text { to }(k+1) / 2 \\
1, i \text { var ies from }(k+3) / 2 \text { to } k
\end{array}
$$$$
f\left(v_{i} w_{i}\right)=0, i \text { var ies from } 1 \text { to }(k+1) / 2
$$$$
1, i \text { var ies from }(k+3) / 2 \text { to } k
$$$$
f\left(w_{i} x_{i}\right)=0, i \text { var ies from } 1 \text { to }(k-1) / 2
$$$$
1, i \text { var ies from }(k+1) / 2 \text { to } k
$$

$f\left(x_{i} u_{i+1}\right)=0, i$ var ies from 1 to $(k-1) / 2$

$$
1, i \text { var ies from }(k+1) / 2 \text { to } k-1
$$

$f\left(u_{2 i} u_{2 i+1}\right)=0, i$ var ies from 1 to $2 k / 2$

$$
1, i \text { var ies from }(2 k+2) / 2 \text { to } k-2
$$

The following table expresses the cardinality of vertices and edges of MSCL for the above graph.

\begin{tabular}{|c|c|c|}
\hline$m$ & 0 & 1 \\
\hline$\left|v_{f}(t)\right|$ & $\frac{5 k}{2}$ & $\frac{5 k}{2}$ \\
\hline$\left|\theta_{f}(t)\right|$ & $3 \mathrm{k}-1$ & $3 \mathrm{k}$ \\
\hline
\end{tabular}

\section{Case (ii) $k$ is even}

$$
\begin{aligned}
& f\left(u_{i}\right)=0, i \text { var ies from } 1 \text { to }(2 k) / 2 \\
& 1, i \text { var ies from }(2 k+2) / 2 \text { to } 2 k \\
& \begin{aligned}
f\left(v_{i}\right)= & 0, i \text { var ies from } 1 \text { to }(k) / 2 \\
& 1, i \text { var ies from }(k+2) / 2 \text { to } k
\end{aligned} \\
& f\left(w_{i}\right)=0, i \text { var ies from } 1 \text { to }(k) / 2 \\
& 1, i \text { var ies from }(k+2) / 2 \text { to } k \\
& f\left(x_{i}\right)=0, i \text { var ies from } 1 \text { to }(k) / 2 \\
& 1, i \text { var ies from }(k+2) / 2 \text { to } k
\end{aligned}
$$

Then the induced edge labeling is as follows

$$
\begin{aligned}
& f\left(u_{i} u_{i+1}\right)=0, i \text { var ies from } 1 \text { to }(2 k) / 2 \\
& 1, i \text { var ies from }(2 k+2) / 2 \text { to } 2 k-1 \\
& f\left(u_{i} v_{i}\right)=0, i \text { var ies from } 1 \text { to }(k) / 2 \\
& 1, i \text { var ies from }(k+2) / 2 \text { to } k \\
& f\left(v_{i} w_{i}\right)=0, i \text { var ies from } 1 \text { to }(k) / 2 \\
& 1, i \text { var ies from }(k+2) / 2 \text { to } k \\
& f\left(w_{i} x_{i}\right)=0, i \text { var ies from } 1 \text { to }(k) / 2 \\
& 1, i \text { var ies from }(k+2) / 2 \text { to } k \\
& f\left(x_{i} u_{i+1}\right)=0, i \text { var ies from } 1 \text { to }(k) / 2 \\
& 1, i \text { var ies from }(k+2) / 2 \text { to } k-1 \\
& f\left(u_{2 i} u_{2 i+1}\right)=0, i \text { var ies from } 1 \text { to } 2 k / 2 \\
& 1, i \text { var ies from }(2 k+2) / 2 \text { to } k-2
\end{aligned}
$$

The following table expresses the cardinality of vertices and edges of MSCL for the above graph.

\begin{tabular}{|c|c|c|}
\hline \multicolumn{1}{|c|}{} & 0 & 1 \\
\hline \multicolumn{1}{|c|}{$\mid v_{f}(t)$} & $\frac{5 k+1}{2}$ & $\frac{5 k-1}{2}$ \\
\hline||$e_{f}(t)$ & $3 \mathrm{k}$ & \\
& $3 \mathrm{k}-1$ & \\
\hline
\end{tabular}

Hence subdivision of a pentagonal snake $\mathrm{S}\left(\mathrm{PS}_{\mathrm{k}}\right)$ admits mean square cordial labeling $\forall \mathrm{k} \geq 2$.

Illustration:Mean square cordial labeling of pentagonal snake:

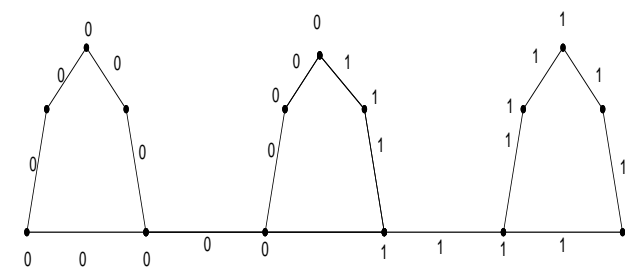

Figure 4:Mean square cordial labeling of pentagonal snake A(PS6)

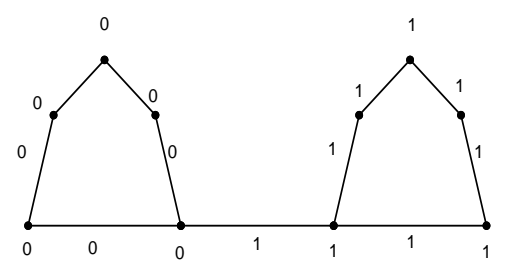

Figure 5:Mean square cordial labeling of pentagonal snake A(PS4) 


\section{CONCLUSION}

In this section mean square cordial labeling is investigated for pentagonal snake graphs. It can be further investigated by the researcher for some more snake related graphs like alternate triangular snake graphs, double triangular snake graphs, alternate quadrilateral snake graphs, double quadrilateral graphs etc.

\section{FUTURE SCOPE}

Graph operations like union, intersection, corona of two graphs etc., can also be discussed for mean square cordial labeling in future.

\section{ACKNOWLEDGEMENTS}

We offer our sincere thanks to the referee for the valuable suggestion for revision of the paper.

\section{REFERENCES}

[1]A. Gallian A Dynamic Survey of Graph Labeling Electronic Journal of Combinatorics, Vol. 18, 2011, pp. 1-219

[2] F. Harary Graph Theory Narosa Publishing House, New Delhi, 1988.

[3]I. Cahit Cordial Graphs: A Weaker Version of Graceful and Harmonious Graphs Ars Combinatoria, Vol. 23, No. 3, 1987, pp. 201-207.

[4]Ponraj, M.Sivakumar and M.Sundaram, Mean cordial labeling of graphs Open journal of Discrete Mathematics, 2(4)(2012), 145-14

[5]A.Nellai Murugan, S.Heerajohn Special Class of Mean Square Cordial Graphs, International Journal of Applied Research 2015; 1(11): $128-131$

[6]A.Nellai Murugan, S.Heerajohn Tree Related Mean Square Cordial Graphs ,outreach IX 2016 126-131 ,A multidisciplinary refereed journal

[7]A.Nellai Murugan, S.Heerajohn Cycle related of Mean Square Cordial Graphs, International Journal of research and development organization.

[8]S.Dhanalakshmi and N.Parvathi Mean square cordial labelling related to some cyclic graphs, International Journal of Engineering and Technology.

[9]S.Dhanalakshmi and N.Parvathi Mean square cordial labelling related to some acyclic graphs and its rough approximations, National Conference on Mathematical Techniques and its Applications (NCMTA 18) IOP Publishing IOP Conf. Series: Journal of Physics: Conf. Series 1000 (2018) 012040 doi :10.1088/1742-6596/1000/1/01204

\section{AUTHORS PROFILE}

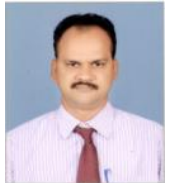

S. Thirunavukkarasu, Assitant Professor, Department of Information Technology, Bharath Institute of Higher Education and Research, Chennai, India. 